\title{
Monitoring coherence between the macro and microcirculation in septic shock
}

\author{
Jan Bakker $r^{\mathrm{a}, \mathrm{b}, \mathrm{c}, \mathrm{d}}$ and Can Ince $\mathrm{a}^{\mathrm{a}}$
}

\begin{abstract}
Purpose of review
Currently, the treatment of patients with shock is focused on the clinical symptoms of shock. In the early phase, this is usually limited to heart rate, blood pressure, lactate levels and urine output. However, as the ultimate goal of resuscitation is the improvement in microcirculatory perfusion the question is whether these currently used signs of shock and the improvement in these signs actually correspond to the changes in the microcirculation.
\end{abstract}

\section{Recent findings}

Recent studies have shown that during the development of shock the deterioration in the macrocirculatory parameters are followed by the deterioration of microcirculatory perfusion. However, in many cases the restoration of adequate macrocirculatory parameters is frequently not associated with improvement in microcirculatory perfusion. This relates not only to the cause of shock, where there are some differences between different forms of shock, but also to the type of treatment.

\section{Summary}

The improvement in macrohemodynamics during the resuscitation is not consistently followed by subsequent changes in the microcirculation. This may result in both over-resuscitation and under-resuscitation leading to increased morbidity and mortality. In this article the principles of coherence and the monitoring of the microcirculation are reviewed.

\section{Keywords}

endpoints, hemodynamics, lactate, perfusion, peripheral perfusion, resuscitation, shock

\section{INTRODUCTION}

Circulatory dysfunction is a frequent reason for admission to the ICU. The principal role of the circulation is to deliver nutrients (oxygen and fuel) to the organs and remove waste product. This is mainly accomplished by the delivery of red blood cells (RBC) into the microcirculation and the passive diffusion of oxygen from the RBCs to the tissue cells. When requirements are not met, first organ function decreases before ultimately failing. Therefore, the goal in treating circulatory dysfunction is to restore adequate perfusion of the microcirculation. The oxygen content [hemoglobin $(\mathrm{Hb})$ and $\mathrm{Hb}$ saturation] are factors that also determine the ultimate amount of oxygen delivered to the organs. However, in this review we will focus on perfusion as the body has limited ability to improve oxygen content acutely. In addition, in response to changes in oxygen demand the first response of the circulatory system is to improve flow. In situations of stress (shock) the system also influences organ blood flow by redirecting flow to more vital organs at the expense of less the perfusion of less vital organs.
The clinical definition of circulatory dysfunction or failure varies and is usually defined by macrohemodynamic parameters like blood pressure (BP), biomarkers in arterial and (central) venous blood and parameters of organ function. Where shock is the worst form of circulatory failure, its definition is not really usable at the bedside [1] so that one has to rely on the same parameters and biomarkers for its recognition. Not only the use of these, sometimes

${ }^{a}$ Department of Intensive Care Adults, Erasmus MC University Medical Center, Rotterdam, The Netherlands, 'Department of Pulmonary and Critical Care, New York University School of Medicine, 'Division of Pulmonary, Allergy, and Critical Care Medicine, Columbia University College of Physicians \& Surgeons, New York, New York, USA and ${ }^{\mathrm{d} D e p a r t m e n t}$ of Intensive Care, Pontificia Universidad Católica de Chile, Santiago, Chile

Correspondence to Jan Bakker, MD, PhD, FCCM, FCCP, Department of Intensive Care Adults, Erasmus MC University Medical Center, PO Box 2040, Room Ne-415, 3000 CA Rotterdam, The Netherlands.

Tel: +31 10 7030772; e-mail: jan.bakker@erasmusmc.nl

Curr Opin Crit Care 2020, 26:267-272

DOI:10.1097/MCC.0000000000000729 


\section{KEY POINTS}

- During the development of shock microcirculatory perfusion parameters follow the deterioration of macrocirculatory symptoms and parameters of shock (coherence).

- Although in some forms of shock coherence is also present in the resuscitation of shock, especially septic shock represents a state in which coherence is lost.

- As the resuscitation is still mainly focused on macrocirculatory parameters, the loss of coherence may result in both over and under-resuscitation.

- Loss of coherence is associated with increased morbidity and mortality.

- Although loss of coherence between the macrocirculation and peripheral perfusion parameters is also present following initial resuscitation in septic shock, limited research have studied the coherence between the microcirculation and peripheral circulation.

neither specific nor sensitive markers, might result in inadequate treatment of the patient.

For this review, we define hemodynamic coherence as the presence of concordance between changes in the macrocirculation and the microcirculation. We will mainly focus on sepsis as this a very frequent cause of circulatory dysfunction in critically ill patients and has been shown to present a both complex microcirculatory dysfunction and a complex response to its treatment.

\section{METHODS TO MONITOR THE MICROCIRCULATION}

The methods available to monitor the microcirculation are numerous but relate to the specific microcirculation of interest. In intact patients this basically means that access to organs is not possible with the exception of patients with an enteral stoma which provides access to gut mucosa. The accessible sites are thus mainly limited to skin and the sublingual area. In different contexts, the mucosa of the rectum and vagina has been used $[2,3]$.

For the skin the methods available have been reviewed extensively earlier $[4,5]$ and will not be discussed here. The sublingual area provides an easy opportunity to visualize a true microcirculatory network. From its first clinically available device, some 20 years ago [6], the technique has been further developed [7], an automated scoring system has become available [8"] and guidelines for the practical use and interpretation have been published [9"]. Although other areas can be used to visualize the microcirculation (nailbed, conjunctive, retina) their clinical use is thus far limited.

\section{THE IMPORTANCE OF COHERENCE}

Where the ultimate goal of resuscitation is to restore microcirculatory perfusion and oxygen exchange, the question is whether the clinically used parameters adequately reflect these processes (Fig. 1) [10]. Perceived adequate macrohemodynamics in the presence of abnormal microcirculatory perfusion has been referred to as microcirculatory shock [11]. In addition, normal peripheral microcirculatory perfusion in patients with septic shock is associated with a significantly lower mortality in the presence of similar macrohemodynamics [12"]. In both cases, the lack of coherence could represent a different (clinical) phenotype but could also result in either over-resuscitation or under-resuscitation of patients. A relevant clinical example of this could be the use of lactate in the early resuscitation of septic shock as recommended by the Surviving Sepsis Campaign Guidelines [13]. In these, fluid administration is recommended in patients with increased lactate levels as these would mark tissue hypoperfusion with the ultimate goal to normalize lactate levels [13]. Both this assumption and the use of normalization have been seriously questioned as lactate may not (always) indicate tissue hypoperfusion and microcirculatory perfusion may be normal when lactate levels have not normalized yet $[14,15]$ and coherence may be lost [16]. Both conditions could lead to over resuscitation with associated risk of increased morbidity and mortality $[17,18]$.

\section{COHERENCE DURING THE DEVELOPMENT OF CIRCULATORY FAILURE}

In acute models of hemorrhagic shock, tamponade, cardiogenic shock and cardiac arrest coherence exists during the development of shock. In other words, during the development of shock, as signaled by the changes in macrocirculatory parameters, also the microcirculation shows abnormal perfusion parameters [19-27]. Although during the development of septic shock, characterized by hypotension and decreased cardiac output (CO), hemodynamic coherence is present $[20,28-30]$. However, sepsis with preserved macrohemodynamics may still show an abnormal microcirculation [31,32]. In contrast to other organs, the microcirculation of the brain seems to be preserved during the development of septic, cardiogenic and hemorrhagic shock [22,33,34] despite significant macrocirculatory abnormalities.

The study of coherence in human models of shock is limited but in models of hypovolemia in 


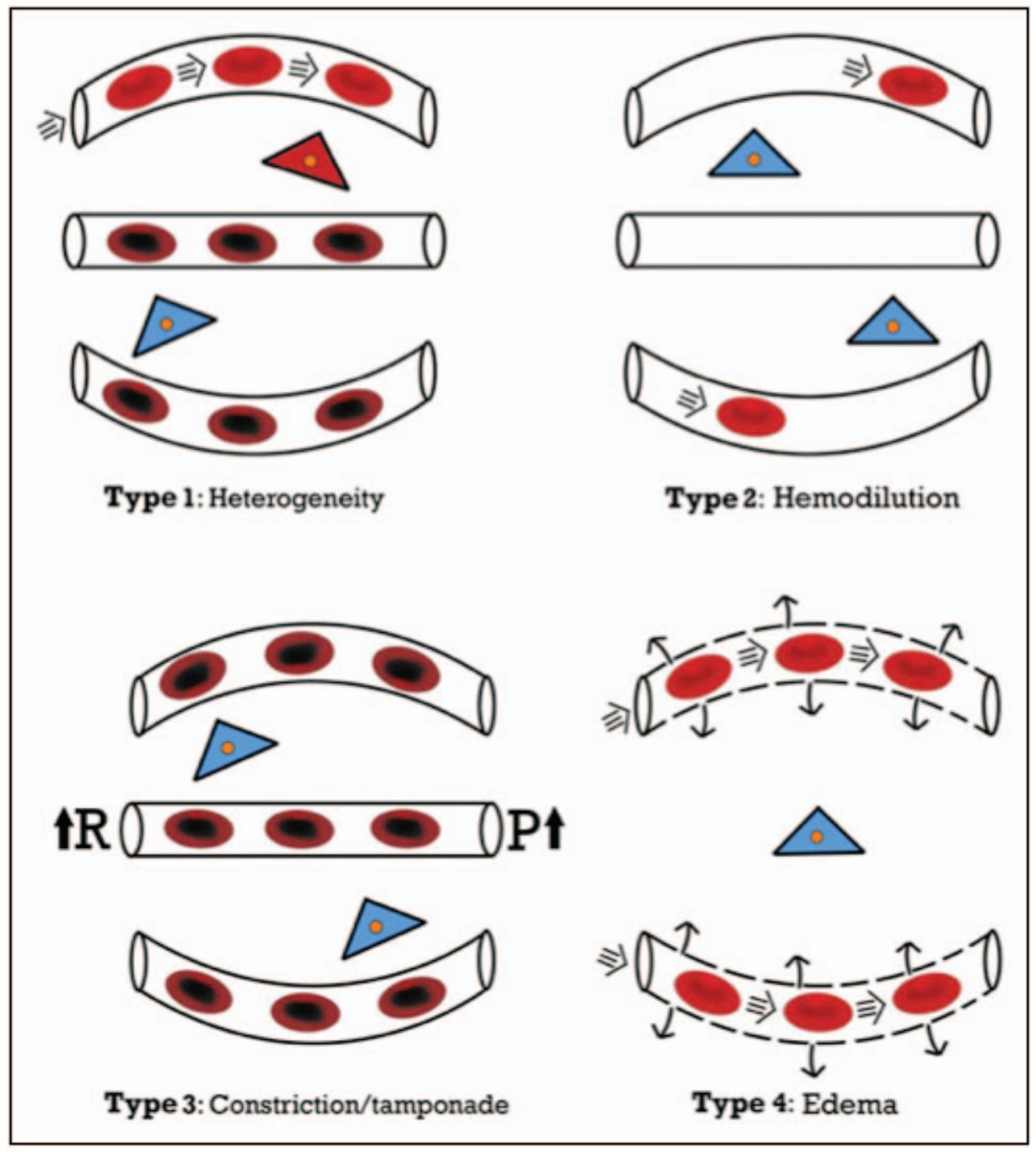

FIGURE 1. Microcirculatory changes in lost hemodynamic coherence. Different microcirculatory changes characterize the mechanisms associated with the loss of hemodynamic coherence resulting in a decreased delivery of oxygen to the cells. Type 1: Heterogenous perfusion of the microcirculation as seen in sepsis. Some capillaries have no flow where others have normal or increased flow resulting in scanted oxygen delivery to some cells. Type 2: Loss of hematocrit in the microcirculation as seen in hemodilution. The result in not only reduced delivery of oxygen but also increased diffusion distance between the red blood cells and the tissue cells. Type 3: Stasis in the microcirculation induced by altered systemic variables. Increased arterial resistance $(R)$, increased venous pressure $(P)$ resulting in a tamponade of the microcirculation. Type 4: Development of tissue edema (e.g. due to capillary leak) resulting in increased diffusion distance between the normally perfusion capillaries and the tissue cells. Red: well oxygenated red blood cells and tissue cells. Dark red/black: red blood cells with decreased oxygen saturation. Blue: tissue cells with reduced oxygenation. From [10].

healthy volunteers, hemodynamic coherence is also present during the progression of circulatory abnormalities. In a model of simulated hypovolemia Bartels et al. [35] showed that lower body negative pressure resulted in decreased CO (while BP was maintained) coinciding with abnormal sublingual microcirculatory perfusion. In human models of sepsis, the (macrohemodynamic) characteristics mimic the ones seen in patients presenting with sepsis [36-39]. In a volunteer study, Draisma et al. [38] showed that a bolus of endotoxin in healthy volunteers resulted in a decrease in mean arterial pressure and an increase in heart rate (HR) characteristic of clinical sepsis. The bolus of endotoxin was also associated with a decrease in vascular reactivity and a decrease in microcirculatory perfusion, both being restored $4 \mathrm{~h}$ after the bolus [38].

With the exception of the brain microcirculation, we can thus assume that in the early phase of severe macrocirculatory dysfunction the microcirculation is compromised. It is conceivable that microcirculatory perfusion abnormalities may 
develop before macrocirculatory hemodynamic changes are prominent. This could especially be the case during a tamponade of the microcirculation by increased central venous pressure (CVP). A clue to this was the finding by Vellinga et al. [40] that patients with increased CVP showed an impaired sublingual microcirculation. In patients with sepsis [41] and cardiac failure [42], CVP has been shown to be an independent risk factor for acute kidney injury despite adequate macrocirculatory parameters. However, all of these findings were postresuscitation and thus, other than keeping CVP as low as possible during resuscitation of shock, general recommendations cannot be made from this [43]. It is clinically not feasible to monitor sublingual microcirculatory perfusion in all patients at risk while having normal macrocirculatory parameters although monitoring by ICU nurses of the sublingual microcirculation using a simplified scoring system has been shown to be feasible [44]. In addition, the benefit of intervening to restore microcirculatory abnormalities in patients with adequate macrocirculatory parameters has not been shown, this clearly has a sound rationale and represents a research challenge. Overall, the assumption that the microcirculation is compromised in patients with developing abnormal macrocirculatory parameters in a relevant context seems valid.

\section{COHERENCE IN THE TREATMENT PHASE: EXPERIMENTAL DATA}

The main clinically relevant phase of the presence or absence of hemodynamic coherence is the resuscitation phase. As argued earlier, the absence of coherence in this phase may have significant effects on the adequacy of resuscitation as it may result in both over-resuscitation and under-resuscitation. It is clear from experimental models in many different pathophysiologic conditions, including sepsis, that resuscitation on global hemodynamic parameters frequently fails to restore microcirculatory perfusion and oxygenation $[25,28,45-47]$. The effects in different microcirculatory systems or even within one microcirculatory system may however not be similar $[30,48]$. There may be few exceptions possibly related to the specific hemodynamic compromise or that may reflect differences in individual responses. In a model of tamponade, van Genderen et al. [20] showed that removing the pericardial fluid resulted in a rapid restoration of both macrocirculatory and microcirculatory perfusion parameters. This was very different in the model of septic shock these researchers studied, where restoration of baseline macrohemodynamics did not restore the microcirculation, only resuscitation to a hyperdynamic state restored the microcirculation. However, as there were no control animals in their study, the effect of time could not be ruled out [20]. Although some (experimental) treatments have shown to be more effective in restoring microcirculatory perfusion and oxygen exchange than the use of fluids only $[46,49,50]$ the type of fluids may not be an unimportant aspect of restoring microcirculatory perfusion as well $[51,52]$. However, a detailed discussion about this is beyond the scope of this publication.

\section{CLINICAL DATA}

Several studies in patients with sepsis and septic shock have shown a lack of coherence between the macrocirculation and microcirculation when treating the patient or following optimization of macrohemodynamics [53-59]. Similar findings have been shown in patients with cardiac failure, cardiogenic shock and hemorrhagic shock [56,60-62]. When there is a lack of coherence following initial resuscitation, some of these studies have shown that recovery of the microcirculation may take a much longer time. In many of these studies, the lack of coherence and prolonged recovery time of the microcirculation has been associated with mortality. Persistent macrohemodynamic abnormalities in combination with microcirculatory abnormalities may impose an even more increased risk of mortality [63].

The relevance of different microcirculations, limited by the scarce availability of these in patients, is unknown. In a study in patient with sepsis two studies reported on the coherence of the intestinal microcirculation $[64,65]$. Both studies showed the absence of coherence between intestinal microcirculation the sublingual microcirculation and the macrohemodynamics. In the study by Boerma et al. [64], it was shown that recovery of coherence between the sublingual and intestinal sites was restored after several days.

The use of vasoactive agents to resuscitate the microcirculation has been reviewed earlier [66] and is outside of the scope of this article. The use of beta blocker therapy has gained new interest as both experimental and clinical studies have shown positive effects on microcirculatory perfusion $[50,67,68]$. As in these studies beta blockers were titrated to HR, the recent finding of the additional effect on mortality of increased HR in patients with abnormal microcirculatory perfusion [63] should encourage additional research in this context.

\section{CONCLUSION}

From the previous the following clinical consequences could be drawn. In patients with abnormal macrohemodynamics it is very likely that microcirculatory 
parameters of perfusion are impaired as well. Although in some clinical contexts the improvement of the macrohemodynamics may coincide with improvements in microcirculatory perfusion, restoration of microcirculatory perfusion by aiming to restore macrocirculatory parameters, especially in patients with severe sepsis and septic shock, is unlikely. If anything, restoration of microcirculatory perfusion may take much more time. The clinical relevance is that the absence of coherence, and thus rapid restoration of microcirculatory perfusion is associated with increased mortality in the majority of studies and clinical contexts. Where specific vasoactive drugs and specific resuscitation fluids have been shown to favor improvements in microcirculatory perfusion both the clinical protocol on how to use these and the subsequent effect on patient outcome has hardly been studied.

Therefore, the ultimate conclusion of this article is a call to design studies that evaluate the early resuscitation of the microcirculation together or following restoration of macrohemodynamics.

\section{Acknowledgements}

None.

\section{Financial support and sponsorship}

None.

\section{Conflicts of interest}

J.B.: none. C.I.: Received honoraria and independent research grants from Fresenius-Kabi, Bad Homburg, Germany; La Jolla Pharmaceutical Co., La Jolla, CA, USA and Cytosorbents Monmouth, NJ, USA. C.I. has developed SDF imaging, which is a hand-held video microscope, and is listed as the inventor on related patents commercialized by MicroVision Medical (MVM) under a license from the Academic Medical Center (AMC). He receives no royalties or benefits from this license. Braedius Medical, a company owned by a relative of C.I., has developed and designed a handheld microscope called CytoCam-IDF imaging. C.I. has no financial relationship with Braedius Medical of any sort, that is, never owned shares, or received consultancy or speaker fees from Braedius Medical. The MicroTools software for automatic analysis of microcirculation images is being developed by Matthias Hilty (MH) with support from Active Medical BV of which C.I. and MPH are shareholders. Active Medical runs an internet site called microcirculationacademy.org which offers educational courses and services related to clinical microcirculation and provides more information regarding MicroTools.

\section{REFERENCES AND RECOMMENDED} READING

Papers of particular interest, published within the annual period of review, have been highlighted as:

- of special interest

- of outstanding interest

1. Cecconi M, De Backer $D$, Antonelli M, et al. Consensus on circulatory shock and hemodynamic monitoring. Task Force of the European Society of Intensive Care Medicine. Intensive Care Med 2014; 40:1795-1815.

2. Dondorp AM, Ince $C$, Charunwatthana $P$, et al. Direct in vivo assessment of microcirculatory dysfunction in severe falciparum malaria. J Infect Dis 2008; 197:79-84.

3. Weber MA, Diedrich CM, Ince C, et al. Focal depth measurements of the vaginal wall: a new method to noninvasively quantify vaginal wall thickness in the diagnosis and treatment of vaginal atrophy. Menopause 2016; 23:833-838.

4. Lima A, Bakker J. Clinical assessment of peripheral circulation. Curr Opin Crit Care 2015; 21:226-231.

5. van Genderen ME, van Bommel J, Lima A. Monitoring peripheral perfusion in critically ill patients at the bedside. Curr Opin Crit Care 2012; 18:273-279.

6. Groner W, Winkelman JW, Harris AG, et al. Orthogonal polarization spectral imaging: a new method for study of the microcirculation. Nat Med 1999; 5:1209-1212.

7. Ocak I, Kara A, Ince C. Monitoring microcirculation. Best Pract Res Clin Anaesthesiol 2016; 30:407-418.

8. Hilty MP, Guerci $\mathrm{P}$, Ince $\mathrm{Y}$, et al. MicroTools enables automated quantification

- of capillary density and red blood cell velocity in handheld vital microscopy. Commun Biol 2019; 2:217.

The clinical application of assessing the sublingual microcirculation at the bedside and also in clinical research has been limited by the labor intensive way of grading the state of the microcirculation. The computer program discussed in this article might chance these barriers by rapidly analyzing the recording a providing a detailed description of the state of the microcirculation.

9. Ince $\mathrm{C}$, Boerma EC, Cecconi M, et al. Second consensus on the assessment

- of sublingual microcirculation in critically ill patients: results from a Task Force of the European Society of Intensive Care Medicine. Intensive Care Med 2018; 44:281-299.

Important expert consensus on the different aspects of recording and analyzing the microcirculation with the current available tools.

10. Ince $\mathrm{C}$. Hemodynamic coherence and the rationale for monitoring the microcirculation. Crit Care 2015; 19(Suppl 3):S8.

11. Kanoore Edul VS, Ince $C$, Dubin $A$. What is microcirculatory shock? Curr Opin Crit Care 2015; 21:245-252.

12. Hernandez G, Kattan E, Ospina-Tascon G, et al. Capillary refill time status

- could identify different clinical phenotypes among septic shock patients fulfilling Sepsis-3 criteria: a post hoc analysis of ANDROMEDA-SHOCK trial. Intensive Care Med 2020; 46:816-818.

The article reports that septic shock patients with abnormal peripheral perfusion that persists after initial resuscitation may represent a different clinical phenotype. This may correspond with the different phenotype of patients with persistent abnormal sublingual microcirculation as both of these groups are characterized by increased morbidity and mortality.

13. Levy MM, Evans LE, Rhodes A. The surviving sepsis campaign bundle: 2018 update. Intensive Care Med 2018; 44:925-928.

14. Hernandez $G$, Luengo $C$, Bruhn $A$, et al. When to stop septic shock resuscitation: clues from a dynamic perfusion monitoring. Ann Intensive Care $2014 ; 4: 30$.

15. Hernandez G, Bellomo R, Bakker J. The ten pitfalls of lactate clearance in sepsis. Intensive Care Med 2019; 45:82-85.

16. Bakker J. Lactate levels and hemodynamic coherence in acute circulatory failure. Best Pract Res Clin Anaesthesiol 2016; 30:523-530.

17. Ince $C$. The rationale for microcirculatory guided fluid therapy. Curr Opin Crit Care 2014; 20:301-308.

18. Spiegel R, Gordon D, Marik PE. The origins of the Lacto-Bolo reflex: the mythology of lactate in sepsis. J Thorac Dis 2020; 12(S1):S48-S53.

19. Arnemann $\mathrm{PH}$, Hessler $M$, Kampmeier $T$, et al. Resuscitation with hydroxyethyl starch maintains hemodynamic coherence in ovine hemorrhagic shock. Anesthesiology 2020; 132:131-139.

20. van Genderen ME, Klijn E, Lima A, et al. Microvascular perfusion as a target for fluid resuscitation in experimental circulatory shock. Crit Care Med 2014; 42:E96-E105.

21. Dubin A, Pozo MO, Ferrara G, et al. Systemic and microcirculatory responses to progressive hemorrhage. Intensive Care Med 2009; 35:556-564.

22. Wan $Z$, Ristagno G, Sun $S$, et al. Preserved cerebral microcirculation during cardiogenic shock. Crit Care Med 2009; 37:2333-2337.

23. Wu CY, Yeh YC, Chien CT, et al. Laser speckle contrast imaging for assessing microcirculatory changes in multiple splanchnic organs and the gracilis muscle during hemorrhagic shock and fluid resuscitation. Microvasc Res 2015; 101:55-61. 
24. Yin $L$, Yang $Z, Y u H$, et al. Changes in sublingual microcirculation is closely related with that of bulbar conjunctival microcirculation in a rat model of cardiac arrest. Shock 2016; 45:428-433.

25. Krupickova $P$, Mlcek $M$, Huptych $M$, et al. Microcirculatory blood flow during cardiac arrest and cardiopulmonary resuscitation does not correlate with global hemodynamics: an experimental study. J Transl Med 2016; 14:163.

26. Fries $\mathrm{M}, \mathrm{Weil} \mathrm{MH}$, Chang $\mathrm{YT}$, et al. Microcirculation during cardiac arrest and resuscitation. Crit Care Med 2006; 34(12 Suppl):S454-S457.

27. Langeland $\mathrm{H}$, Lyng $\mathrm{O}$, Aadahl $\mathrm{P}$, et al. The coherence of macrocirculation, microcirculation, and tissue metabolic response during nontraumatic hemorrhagic shock in swine. Physiol Rep 2017; 5:e13216.

28. Legrand $M$, Bezemer $R$, Kandil $A$, et al. The role of renal hypoperfusion in development of renal microcirculatory dysfunction in endotoxemic rats. Intensive Care Med 2011; 37:1534-1542.

29. Fang $X$, Tang $W$, Sun $S$, et al. Comparison of buccal microcirculation between septic and hemorrhagic shock. Crit Care Med 2006; 34(12 Suppl):S447-S453.

30. Dubin A, Edul VS, Pozo MO, et al. Persistent villi hypoperfusion explains intramucosal acidosis in sheep endotoxemia. Crit Care Med 2008; 36: $535-542$.

31. Lam C, Tyml K, Martin C, et al. Microvascular perfusion is impaired in a rat model of normotensive sepsis. J Clin Invest 1994; 94:2077-2083.

32. Farquhar I, Martin CM, Lam C, et al. Decreased capillary density in vivo in bowel mucosa of rats with normotensive sepsis. J Surg Res 1996; 61:190-196.

33. Wan Z, Sun S, Ristagno G, et al. The cerebral microcirculation is protected during experimental hemorrhagic shock. Crit Care Med 2010; 38:928-932.

34. Rosengarten $B$, Hecht M, Auch $D$, et al. Microcirculatory dysfunction in the brain precedes changes in evoked potentials in endotoxin-induced sepsis syndrome in rats. Cerebrovasc Dis 2007; 23:140-147.

35. Bartels SA, Bezemer R, Milstein DM, et al. The microcirculatory response to compensated hypovolemia in a lower body negative pressure model. Microvasc Res $2011 ; 82: 374-380$.

36. Suffredini AF, Fromm RE, Parker MM, et al. The cardiovascular response of normal humans to the administration of endotoxin. N Engl J Med 1989; 321:280-287.

37. Kiers D, Koch RM, Hamers L, et al. Characterization of a model of systemic inflammation in humans in vivo elicited by continuous infusion of endotoxin. Sci Rep 2017; 7:40149.

38. Draisma A, Bemelmans R, van der Hoeven JG, et al. Microcirculation and vascular reactivity during endotoxemia and endotoxin tolerance in humans. Shock 2009; 31:581-585.

39. van Loon LM, Stolk RF, van der Hoeven JG, et al. Effect of vasopressors on the macro- and microcirculation during systemic inflammation in humans in vivo. Shock 2020; 53:171-174.

40. Vellinga NA, Ince C, Boerma EC. Elevated central venous pressure is associated with impairment of microcirculatory blood flow in sepsis: a hypothesis generating post hoc analysis. BMC Anesthesiol 2013; 13:17.

41. Legrand $M$, Dupuis $C$, Simon $C$, et al. Association between systemic hemodynamics and septic acute kidney injury in critically ill patients: a retrospective observational study. Crit Care 2013; 17:R278.

42. van den Akker JPC, Bakker J, Groeneveld ABJ, et al. Risk indicators for acute kidney injury in cardiogenic shock. J Crit Care 2019; 50:11-16.

43. Chen $X$, Wang $X$, Honore $P M$, et al. Renal failure in critically ill patients, beware of applying (central venous) pressure on the kidney. Ann Intensive Care 2018; 8:91

44. Tanaka $S$, Harrois $A$, Nicolai $C$, et al. Qualitative real-time analysis by nurses of sublingual microcirculation in intensive care unit: the MICRONURSE study. Crit Care 2015; 19:388.

45. Ergin B, Zafrani L, Kandil A, et al. Fully balanced fluids do not improve microvascular oxygenation, acidosis and renal function in a rat model of endotoxemia. Shock 2016; 46:83-91.

46. Guerci $P$, Ergin B, Kapucu A, et al. Effect of polyethylene-glycolated carboxyhemoglobin on renal microcirculation in a rat model of hemorrhagic shock. Anesthesiology 2019; 131:1110-1124.

47. Siegemund M, van Bommel J, Stegenga ME, et al. Aortic cross-clamping and reperfusion in pigs reduces microvascular oxygenation by altered systemic and regional blood flow distribution. Anesth Analg 2010; 111:345-353.
48. Siegemund $M$, Van Bommel J, Sinaasappel $M$, et al. The NO donor SIN-1 improves intestinal-arterial $\mathrm{P}(\mathrm{CO}(2))$ gap in experimental endotoxemia: an animal study. Acta Anaesthesiol Scand 2007; 51:693-700.

49. Zafrani L, Ergin B, Kapucu A, et al. Blood transfusion improves renal oxygenation and renal function in sepsis-induced acute kidney injury in rats. Crit Care 2016; 20:406.

50. Jacquet-Lagreze $M$, Allaouchiche $B$, Restagno D, et al. Gut and sublingual microvascular effect of esmolol during septic shock in a porcine model. Crit Care 2015; 19:241.

51. Wu CY, Chan KC, Cheng YJ, et al. Effects of different types of fluid resuscitation for hemorrhagic shock on splanchnic organ microcirculation and renal reactive oxygen species formation. Crit Care 2015; 19:434.

52. van Bommel J, de Korte D, Lind A, et al. The effect of the transfusion of stored RBCs on intestinal microvascular oxygenation in the rat. Transfusion (Paris) $2001 ; 41: 1515-1523$.

53. De Backer D, Donadello K, Sakr $\mathrm{Y}$, et al. Microcirculatory alterations in patients with severe sepsis: impact of time of assessment and relationship with outcome. Crit Care Med 2013; 41:791-799.

54. Edul VS, Enrico C, Laviolle B, et al. Quantitative assessment of the microcirculation in healthy volunteers and in patients with septic shock. Crit Care Med 2012; 40:1443-1448.

55. Trzeciak S, McCoy JV, Phillip Dellinger R, et al. Early increases in microcirculatory perfusion during protocol-directed resuscitation are associated with reduced multiorgan failure at $24 \mathrm{~h}$ in patients with sepsis. Intensive Care Med 2008; 34:2210-2217.

56. Tachon G, Harrois A, Tanaka $S$, et al. Microcirculatory alterations in traumatic hemorrhagic shock. Crit Care Med 2014; 42:1433-1441.

57. LeDoux D, Astiz ME, Carpati CM, et al. Effects of perfusion pressure on tissue perfusion in septic shock. Crit Care Med 2000; 28:2729-2732.

58. Hernandez G, Boerma EC, Dubin A, et al. Severe abnormalities in microvascular perfused vessel density are associated to organ dysfunctions and mortality and can be predicted by hyperlactatemia and norepinephrine requirements in septic shock patients. J Crit Care 2013; 28: 538.e9-538.e14.

59. Sakr $Y$, Dubois MJ, De Backer D, et al. Persistent microcirculatory alterations are associated with organ failure and death in patients with septic shock. Crit Care Med 2004; 32:1825-1831.

60. De Backer D, Creteur J, Dubois MJ, et al. Microvascular alterations in patients with acute severe heart failure and cardiogenic shock. Am Heart J 2004; 147:91-99.

61. den Uil CA, Lagrand WK, van der Ent M, et al. Conventional hemodynamic resuscitation may fail to optimize tissue perfusion: an observational study on the effects of dobutamine, enoximone, and norepinephrine in patients with acute myocardial infarction complicated by cardiogenic shock. PLoS One 2014; 9:e103978.

62. Chommeloux J, Montero S, Franchineau G, et al. Microcirculation evolution in patients on venoarterial extracorporeal membrane oxygenation for refractory cardiogenic shock. Crit Care Med 2020; 48:e9-e17.

63. Vellinga NA, Boerma EC, Koopmans $M$, et al. International study on microcirculatory shock occurrence in acutely ill patients. Crit Care Med 2015; $43: 48-56$

64. Boerma EC, van der Voort PH, Spronk PE, et al. Relationship between sublingual and intestinal microcirculatory perfusion in patients with abdominal sepsis. Crit Care Med 2007; 35:1055-1060.

65. Edul VS, Ince C, Navarro N, et al. Dissociation between sublingual and gut microcirculation in the response to a fluid challenge in postoperative patients with abdominal sepsis. Ann Intensive Care 2014; 4:39.

66. Boerma EC, Ince $C$. The role of vasoactive agents in the resuscitation of microvascular perfusion and tissue oxygenation in critically ill patients. Intensive Care Med 2010; 36:2004-2018.

67. Morelli A, Donati A, Ertmer C, et al. Microvascular effects of heart rate control with esmolol in patients with septic shock: a pilot study. Crit Care Med 2013; 41:2162-2168

68. Hernandez G, Tapia P, Alegria L, et al. Effects of dexmedetomidine and esmolol on systemic hemodynamics and exogenous lactate clearance in early experimental septic shock. Crit Care 2016; 20:234. 